\title{
UNIFORM AND SOBOLEV EXTENSION DOMAINS
}

\author{
DAVID A. HERRON AND PEKKA KOSKELA
}

(Communicated by Clifford J. Earle, Jr.)

\begin{abstract}
We prove that if a domain $D \subset \mathbf{R}^{n}$ is quasiconformally equivalent to a uniform domain, then $D$ is an extension domain for the Sobolev class $W_{n}^{1}$ if and only if $D$ is locally uniform. We provide examples which suggest that this result is best possible. We exhibit a list of equivalent conditions for domains quasiconformally equivalent to uniform domains, one of which characterizes the quasiconformal homeomorphisms between uniform and locally uniform domains.
\end{abstract}

\section{INTRODUCTION}

This article concerns three classes of domains $D$ in Euclidean $n$-space $\mathbf{R}^{n}$. We call $D$ a $W_{p}^{1}$-extension domain if there exists a bounded linear extension operator from $W_{p}^{1}(D)$ to $W_{p}^{1}\left(\mathbf{R}^{n}\right)$; here $p \geq 1$ and $W_{p}^{1}(D)$ denotes the Sobolev space of measurable functions $u: D \rightarrow \mathbf{R} \cup\{-\infty, \infty\}$ satisfying

$$
\|u\|=\left(\int_{D}|u|^{p}\right)^{1 / p}+\left(\int_{D}|\nabla u|^{p}\right)^{1 / p}<\infty,
$$

where $\nabla u$ represents the distributional gradient of $u$.

O. Martio and J. Sarvas [MS] introduced the notion of a uniform domain; see $[\mathrm{HK}]$ and the references mentioned there for properties of this important class of domains. We call $D$ c-uniform provided each pair of points $x, y \in D$ can be joined by a rectifiable arc $\gamma \subset D$ satisfying

$$
\left\{\begin{array}{l}
l(\gamma) \leq c|x-y| \text { and } \\
\min \left\{l\left(\gamma^{\prime}\right), l\left(\gamma^{\prime \prime}\right)\right\} \leq c \operatorname{dist}(z, \partial D) \quad \text { for all } z \in \gamma .
\end{array}\right.
$$

Here $l(\gamma)$ is the Euclidean arclength of $\gamma$ and $\gamma^{\prime}, \gamma^{\prime \prime}$ are the components of $\gamma \backslash\{z\}$. Condition (1) asserts that the length of $\gamma$ is comparable to the distance between its endpoints and that away from its endpoints $\gamma$ stays away from the boundary $\partial D$ of $D$. In particular, (1) implies that points can be joined in $D$ with a curvilinear double cone which is not too crooked nor too thin.

Received by the editors August 6, 1990.

1980 Mathematics Subject Classification (1985 Revision). Primary 30C60; Secondary 46E35.

Key words and phrases. Sobolev extension domain, uniform domain, locally uniform domain, quasiconformal homeomorphism, quasisymmetry.

Both authors thank the Institut Mittag-Leffler for its hospitality. The first author was partially supported by the University of Cincinnati's Taft Memorial Fund and the second author by the Institut. 
Next, $D$ is $(c, r)$-locally uniform if points $x, y \in D$ with $|x-y| \leq r$ can be joined by a rectifiable arc $\gamma \subset D$ satisfying (1). Every uniform domain is locally uniform; the converse holds for domains $D$ with $\operatorname{diam}(\partial D)<\infty$ [HK, 3.4]. P. W. Jones introduced the class of locally uniform domains and established the following fundamental result relating them and $W_{p}^{1}$-extension domains $[\mathrm{J} 1$, Theorems 1, 3].

Fact. $A(c, r)$-locally uniform domain $D \subset \mathbf{R}^{n}$ is a $W_{p}^{1}$-extension domain for all $p \geq 1$ and the norm of the extension operator is bounded by a constant which depends only on $c, r, p, n$, and $\operatorname{diam}(D)$. Conversely, a finitely connected $W_{2}^{1-}$ extension domain $D \subset \mathbf{R}^{2}$ is locally uniform.

This yields no information about infinitely connected $W_{2}^{1}$-extension domains in $\mathbf{R}^{2}$ and nothing for $W_{n}^{1}$-extension domains in $\mathbf{R}^{n}$. Recently we verified that certain infinitely connected plane domains are $W_{2}^{1}$-extension domains if and only if they are locally uniform [HK, 7.11]. Previously Koskela established numerous properties of $W_{p}^{1}$-extension domains $[\mathrm{K}, \S 6]$.

As every finitely connected plane domain is conformally equivalent to a domain whose boundary components are circles or points, the following generalizes Jones' theorem. Koskela proved this for the case where $\operatorname{diam}(\partial D)<\infty$ [K, 6.3(ii)], but neither his technique nor Jones' can be used to establish our result.

Theorem. Suppose $D \subset \mathbf{R}^{n}$ is quasiconformally equivalent to a uniform domain. Then $D$ is a $W_{n}^{1}$-extension domain if and only if $D$ is locally uniform. Moreover, all constants depend only on the given data.

We explain our proof in $\S 3$ and exhibit examples in $\S 4$ which illustrate why this theorem is essentially best possible. $\S 2$ contains preliminary information, some of which may be of independent interest. In $\S 5$ we present a list of equivalent conditions for certain domains, one of which characterizes the quasiconformal homeomorphisms of locally uniform domains onto uniform domains.

Our notation and terminology conform with that of [HK]. In particular, $B(x, r)$ and $S(x, r)=\partial B(x, r)$ are the open ball and sphere of radius $r$ centered at $x$. We write $c=c(a, \ldots)$ to indicate that $c$ depends only on the parameters $a, \ldots$.

\section{Preliminaries}

Here we collect some facts used in our proof. First we state the following obvious sufficient condition for local uniformity; see the proofs of [HK, 3.7, 7.1].

2.0. Fact. Suppose there exist constants $c, r$ such that for each $z \in \partial D \backslash\{\infty\}$ there is a c-uniform domain $G$, with $D \cap B(z, r) \subset G \subset D$. Then $D$ is $(b, t)$ locally uniform where $b, t$ depend only on $c, r$.

Next we list certain properties of uniform domains: a geometric localization result due to $\mathrm{P}$. W. Jones [J2] and a consequence of work of P. Tukia and J. Väisälä [TV, 2.8, 2.9, 2.15], [V, 3.2, 4.11].

2.1. Fact. Suppose $D \subset \mathbf{R}^{n}$ is c-uniform.

(a) There exists a constant $d=d(c, n)$ such that for each $z \in \partial D \backslash\{\infty\}$ 
and for all $t>0$, there is a d-uniform domain $G$ with $D \cap B(z, t / d) \subset$ $G \subset D \cap B(z, t)$.

(b) If $g: D \rightarrow D^{\prime}$ is a homeomorphism and there exists a constant $h$ such that $|g(u)-g(v)| \leq h|g(w)-g(v)|$ for all $u, v, w \in D$ with $|u-v| \leq|w-v|$, then $D^{\prime}$ is b-uniform where $b=b(c, h, n)$.

The only property of $W_{n}^{1}$-extension domains we require for our proof is the following geometric condition [K, 5.7, 5.8, 5.10].

2.2. Fact. Suppose $D \subset \mathbf{R}^{n}$ is a $W_{n}^{1}$-extension domain. Then there exist constants $a$ and $\rho$, depending only on $n$ and the norm of the extension operator, such that

$$
\left\{\begin{array}{l}
\text { for all } x \in \mathbf{R}^{n} \text { and all } 0<r \leq \rho, \text { points in } D \cap \bar{B}(x, r) \\
(\text { respectively, } D \backslash B(x, r)) \text { can be joined by a continuum in } \\
D \cap \bar{B}(x, \text { ar }) \text { (respectively, } D \backslash B(x, r / a)) .
\end{array}\right.
$$

In a forthcoming paper we generalize a result of $\mathrm{F}$. W. Gehring and O. Martio [GM, 3.1] which has the following corollary. Here $C(f, x)$ denotes the cluster set of $f$ at $x$.

2.3. Fact. Let $f: D \rightarrow D^{\prime}$ be $K$-quasiconformal. Suppose $D \subset \mathbf{R}^{n}$ satisfies (2) and $D^{\prime} \subset \mathbf{R}^{n}$ is c-uniform. Then:

(a) $f^{-1}$ has a continuous extension to $\bar{D}^{\prime}$ and $f$ has a continuous one-toone extension to $\bar{D} \backslash\{\infty\}$.

(b) Assume that either $D$ and $D^{\prime}$ are bounded or that $\infty \in \partial D \cap C(f, \infty)$. Then for each $k>0$ there is an $h=h(k, a, c, K, n)>1$ such that for all $u, v, w \in D$ we have $|f(u)-f(v)| \geq k|f(w)-f(v)|$ whenever $\operatorname{diam}\{u, v, w\} \leq \rho$ and $|u-v| \geq h|w-v|$.

\section{Proof of theOREM}

It suffices to verify that a $W_{n}^{1}$-extension domain quasiconformally equivalent to a uniform domain is in fact locally uniform.

Fix $D \subset \mathbf{R}^{n}$ and suppose there exists a linear extension operator from $W_{n}^{1}(D)$ to $W_{n}^{1}\left(\mathbf{R}^{n}\right)$ with norm $N$. Assume $D^{\prime}$ is $c$-uniform and $f: D \rightarrow D^{\prime}$ is $K$ quasiconformal. We demonstrate that $D$ is $(b, r)$-locally uniform where $b=$ $b(N, K, c, n), r=r(N, K, c, n)$. By 2.0 it suffices to exhibit such constants $b, r$ which enjoy the property that for each $z \in \partial D \backslash\{\infty\}$ there exists a $b$ uniform domain $G$ satisfying

$$
D \cap B(z, r) \subset G \subset D .
$$

Using 2.2 we get constants $a=a(N, n)$ and $\rho=\rho(N, n)$ so that (2) holds. According to $[K, 6.3]$ and the Möbius invariance of uniform domains, we can assume that $\infty \in \partial D \cap \partial D^{\prime}$ and $f^{-1}(\infty)=\infty$. We are now in position to take advantage of 2.3 .

Let $r=\rho / 3 h$ where $h=h(N, K, c, n) \geq 1$ is obtained via $2.3(\mathrm{~b})$ using $k=d$, and $d=d(c, n)$ comes from $2.1(\mathrm{a})$ applied to $D^{\prime}$. Fix $z \in \partial D \backslash\{\infty\}$ and let $z^{\prime}=f(z)$. Employing 2.3(a) we see that $z^{\prime} \neq \infty$ and that

$$
t=\operatorname{dist}\left(z^{\prime}, f(\bar{D} \cap S(z, \rho / 2))\right)>0 \text {. }
$$


Then 2.1(a) ensures the existence of a $d$-uniform domain $G^{\prime}$ satisfying

$$
D^{\prime} \cap B\left(z^{\prime}, t / d\right) \subset G^{\prime} \subset D^{\prime} \cap B\left(z^{\prime}, t\right) .
$$

Next, from 2.3(b) and 2.1(b) we conclude that $G=f^{-1}\left(G^{\prime}\right)$ is $b$-uniform with $b=b(N, K, c, n)$. It remains to verify (3).

Fix $y^{\prime}=f(y) \in S\left(z^{\prime}, t\right) \cap f(\bar{D} \cap S(z, \rho / 2))$. Let $x \in D \cap B(z, r)$ and set $x^{\prime}=f(x)$. Then

whence by $2.3(\mathrm{~b})$

$$
|y-z|=\rho / 2>h r \geq h|x-z|,
$$

$$
t=\left|y^{\prime}-z^{\prime}\right|>d\left|x^{\prime}-z^{\prime}\right|
$$

thus $x^{\prime} \in D^{\prime} \cap B\left(z^{\prime}, t / d\right) \subset G^{\prime}$, so $x \in G$ and (3) holds.

\section{EXAMPLES}

We begin by pointing out that there are simply connected plane domains which are $W_{p}^{1}$-extension domains for each $p>2$ or for each $1 \leq p<2$, yet are not locally uniform; $[\mathrm{M}, 1.5 .2],[\mathrm{K}, 2.5,6.8]$. Furthermore, one cannot replace the quasiconformal equivalence with topological equivalence. We construct explicit examples of topological balls in $\mathbf{R}^{n}(n \geq 3)$ which are $W_{p}^{1}$ extension domains for all $p \geq 1$ but fail to be locally uniform; in fact, according to Jones [J1, p. 75] there are even such Jordan domains. Next, we give examples which indicate that quasiconformal equivalence to a uniform domain is necessary; one cannot weaken this, e.g., to quasiconformal equivalence to a locally uniform domain. Thus, the three hypotheses in our theorem are essential: one must assume $p=n$ and some kind of smoothness criterion is necessary, but quasiconformal equivalence to anything weaker than a uniform domain will not suffice.

4.1. Example. For $n \geq 3$ there exist domains in $\mathbf{R}^{n}$, which are homeomorphic to a ball and are $W_{p}^{1}$-extension domains for all $p \geq 1$, but are not locally uniform.

For notational ease we assume $n=3$; the modifications necessary for general $n \geq 3$ are clear. Let $D=Q \backslash \bigcup I_{j}$ where $Q=(0,2) \times(-1,1) \times(0,2)$ and $I_{j}=\{(1 / j, 0, t): 0 \leq t \leq 1\} \quad(j=1,2, \ldots)$. Then $D$ is homeomorphic to a ball. Next, as $\bigcup I_{j}$ has zero 2-measure, each $u \in W_{p}^{1}(D)$ can be considered as an element of $W_{p}^{1}(Q)$ (simply take an ACL representative for $u$ and extend it to be zero on $\bigcup I_{j}$ ); since $Q$ is an extension domain, so is $D$. Finally, $D$ is not locally uniform because for any $m>0$ we can find points $x=(\varepsilon, \varepsilon, 1 / 2), y=(\varepsilon,-\varepsilon, 1 / 2)$ in $D$ with $|x-y| \leq 1 / m$ such that for any arc $\gamma \subset D$ joining $x, y$, either $l(\gamma)>m|x-y|$ or $l(\alpha)>m \operatorname{dist}(z, \partial D)$ for some $z \in \gamma$, and both components $\alpha$ of $\gamma \backslash\{z\}$; see 4.3 and 4.4 for similar details.

4.2. Proposition. There exist domains $D$ quasiconformally equivalent to locally uniform domains which are $W_{p}^{1}$-extension domains for all $p \geq 1$ but fail to be locally uniform.

We prove 4.2 by presenting two examples of a domain $D$ possessing the following properties.

(a) $D=\phi\left(\mathbf{R}^{n} \backslash Z\right)$ where $\phi$ is a quasiconformal self-homeomorphism of 
$\overline{\mathbf{R}}^{n}$, and $Z$ is the set of all points $\left(x_{1}, \ldots, x_{n-1}, 0\right) \in \mathbf{R}^{n}$ with each $x_{j}$ an integer $(j=1, \ldots, n-1)$.

(b) $D$ satisfies (2) with $a=1, \rho=\infty$.

(c) $D$ is a $W_{p}^{1}$-extension domain for all $p \geq 1$.

(d) $D$ is not locally uniform.

Note that $\mathbf{R}^{n} \backslash Z$ is locally uniform. As the complement of a domain $D$ satisfying (a) has $(n-1)$-measure zero, (c) follows just as in 4.1 ; (b) is obvious, and we indicate why (d) is true. Obviously it is not essential that $Z$ be such a simple set; in fact, according to [HK, 7.4] we could, e.g., replace $Z$ by a union of closed balls each of radius $1 / 3$ with centers at the integer lattice points.

Our first example enjoys the property that $\phi$ is a Möbius transformation. Our second example is of interest because $\phi$ fixes the point at infinity, so $\phi$ is quasisymmetric in the sense of [TV]. For notational clarity we take $n=2$ and identify $\mathbf{R}^{2}$ with $\mathbf{C}$.

4.3. Example. Let $\phi$ be inversion in the unit circle. Then $D=\phi(\mathbf{C} \backslash \mathbf{Z})=$ $\mathbf{C} \backslash\{0, \pm 1, \pm 1 / 2, \pm 1 / 3, \ldots\}$ satisfies (a)-(d) above.

To see that $D$ is not locally uniform, let $m$ be a positive integer and suppose that $\gamma \subset D$ is an arc joining the points $z=i / m^{2}$ and $w=\bar{z}$ with $l(\gamma) \leq$ $m|z-w|$. Fix $x \in \gamma \cap \mathbf{R}$. Then $2|x| \leq l(\gamma) \leq 2 / m$, so $x \in(-1 / m, 1 / m)$. Choose a positive integer $k$ so that $1 /(k+1)<|x|<1 / k$. Then $k \geq m$ and $\operatorname{dist}(x, \partial D) \leq 1 / k(k+1)$, whence

$$
l(\alpha) \geq|x|>1 /(k+1) \geq k \operatorname{dist}(x, \partial D) \geq m \operatorname{dist}(x, \partial D)
$$

for either component $\alpha$ of $\gamma \backslash\{x\}$.

4.4. Example. Let $\phi(z)=z|z|^{-1 / 2}$. Then $D=\phi(\mathbf{C} \backslash \mathbf{Z})$ satisfies (a)-(d) above.

Again, it suffices to show that $D$ fails to be locally uniform. To this end, let $m$ be any positive integer and set $z=2 m+i, w=\bar{z}$. Suppose that $\gamma$ is any arc joining $z, w$ in $D$ with $l(\gamma) \leq m|z-w|=2 m$. Fix $x \in \gamma \cap \mathbf{R}$. Then $2|x-z| \leq l(\gamma) \leq 2 m$ and hence $x \geq m$. Now for integers $j \geq m^{2}$ we have

$$
|\phi(j+1)-\phi(j)| \leq 1 / m, \quad \text { so } \operatorname{dist}(x, \partial D) \leq 1 / 2 m \text {. }
$$

Hence

$$
l(\alpha) \geq 1 \geq m \operatorname{dist}(x, \partial D)
$$

for either component $\alpha$ of $\gamma \backslash\{x\}$.

\section{Comments}

We refer to [GM, HK, K, V] for the definitions of certain terminology used below.

A careful examination of our proof reveals that the essential ingredients are the 'local weak quasisymmetry' property described in $2.3(\mathrm{~b})$ and the 'local uniformity' property expressed in 2.1 (a). Consequently we obtain the following list of equivalent descriptions for certain domains; a detailed proof will be supplied in a forthcoming paper.

Corollary. Suppose $D$ is quasiconformally equivalent to a uniform domain. Then the following are equivalent.

(a) D is a locally uniform domain. 
(b) $D$ is a $W_{p}^{1}$-extension domain for all $p \geq 1$.

(c) $D$ is a $W_{n}^{1}$-extension domain.

(d) There exist constants $a, \rho$ so that $D$ satisfies (2).

(e) There exists a homeomorphism $f$ of $D$ onto a uniform domain and constants $h>1, k>1$ and $\rho$ such that

$$
\left\{\begin{array}{l}
|f(u)-f(v)| \geq k|f(w)-f(v)| \text { whenever } u, v, w \in D \\
\text { and } \operatorname{diam}\{u, v, w\} \leq \rho,|u-v| \geq h|w-v|
\end{array}\right.
$$

Remarks. (a) Notice that $W_{n}^{1}$-extension domains which are quasiconformally equivalent to uniform domains are in fact $W_{p}^{1}$-extension domains for all $p \geq 1$. Also, it turns out that (4) characterizes the quasiconformal homeomorphisms of locally uniform domains onto uniform domains.

(b) Condition (2) is a weak version of F. W. Gehring's linear local connectivity (LLC) [GM, p. 186]. Clearly our theorem remains valid when " $D$ is a $W_{n}^{1}$ extension domain" is replaced by " $D$ satisfies (2) for some $a, \rho$."

(c) Jones [J2] actually proved that 2.1(a) holds for $(c, r)$-locally uniform domains, however in this situation one obtains $G$ only for $0<t<r$. Thus 2.0 characterizes local uniformity.

(d) From our examples we observe that the hypothesis " $D$ is quasiconformally equivalent to a uniform domain" in the corollary cannot be replaced, e.g., by " $D$ is quasiconformally equivalent to a locally uniform domain," even if we replace any of (b), (c), (d) by the stronger conditions that $D$ is an LLC, a QED, or an $L_{n}^{1}$-extension domain.

(e) Condition (4) is a local version of P. Tukia and J. Väisälä's notion of a weak-quasisymmetry [TV, p. 98]. In fact, (4) implies that $f^{-1}$ is locally weakly quasisymmetric. However, there exist homeomorphisms which satisfy (4) whose inverses are not weakly quasisymmetric; e.g., map an infinite cylinder quasiconformally onto a half-space-by [TV, 2.16] and [V, 3.2, 4.11] such a quasiconformal homeomorphism cannot have a weakly quasisymmetric inverse.

\section{REFERENCES}

[GM] F. W. Gehring and O. Martio, Quasiextremal distance domains and extendability of quasiconformal mappings, J. Analyse Math. 45 (1985), 181-206.

[HK] D. A. Herron and P. Koskela, Uniform, Sobolev extension and quasiconformal circle domains, J. Analyse Math. (to appear).

[J1] P. W. Jones, Quasiconformal mappings and extendability of functions in Sobolev spaces, Acta Math. 147 (1981), 71-88.

[J2] - A geometric localization theorem, Adv. in Math. 46 (1982), 71-79.

[K] P. Koskela, Capacity extension domains, Ann. Acad. Sci. Fenn. Ser. A I Math. Dissertationes 73 (1990), 1-42.

[MS] O. Martio and J. Sarvas, Injectivity theorems in plane and space, Ann. Acad. Sci. Fenn. Ser. A I Math. 4 (1978/79), 383-401.

[M] V. G. Maz'ja, Sobolev spaces, Springer-Verlag, Berlin, Heidelberg, New York, and Tokyo, 1985.

[TV] P. Tukia and J. Väisälä, Quasisymmetric embeddings of metric spaces, Ann. Acad. Sci. Fenn. Ser. A I Math. 5 (1980), 97-114.

[V] J. Väisälä, Quasimöbius maps, J. Analyse Math. 44 (1984/85), 218-234. 
InStitut Mittag-Leffler, Auravägen 17 S-182 62 , Djursholm, Sweden

Current address: Department of Mathematics, University of Cincinnati, Cincinnati, Ohio 452210025

E-mail address: herron@ucbeh.bitnet

DePARTMENT OF MATHEMATICS, UNIVERSITY OF JYVÄSKYLÄ, 40100 JYVÄSKyLÄ, FinLAND E-mail address: pkoskela@finjyu.bitnet 